\title{
ON SINGLETONS AND ADJACENCIES OF SET PARTITIONS
}

\author{
AUGUSTINE O. MUNAGI
}

\begin{abstract}
The number of singleton blocks in all partitions of a set $\left\{a_{1}, \ldots, a_{n}\right\}$ is known to be equal to the number adjacencies, that is, pairs of consecutively numbered elements $\left(a_{i}, a_{i+1}\right)$ in a block. We give a generalization of this relation by introducing the $d$-adjacency which is a pair of elements $\left(a_{i}, a_{j}\right)$ satisfying $j-i=d>0$. It is proved that the number of $d$-adjacencies in all partitions is independent of $d$. Then we show that the number of $d$-adjacencies in noncrossing partitions is a function of $d$ by means of an exact formula.
\end{abstract}

1. Adjacencies and singletons. A partition of a set of $n$ distinguishable objects, $A_{n}=\left\{a_{1}, a_{2}, \ldots, a_{n}\right\}$, is a decomposition of $A_{n}$ into nonempty subsets called blocks. The blocks are usually arranged in standard order, that is, in increasing order of least label-numbers.

The number of partitions of $A_{n}$ into $k$ blocks is the Stirling number of the second kind, $S(n, k)$, while the Bell numbers $B_{n}$ are defined by $B_{n}=\sum_{k} S(n, k)$. These numbers may be computed using the formula (see for example [3]),

$$
S(n, k)=\frac{1}{k !} \sum_{j=0}^{k}(-1)^{k-j}\left(\begin{array}{l}
k \\
j
\end{array}\right) j^{n} .
$$

For any positive integer $d$, a (circular) $d$-adjacency is the occurrence of an ordered pair of elements $\left(a_{i}, a_{j}\right)$ in a block such that $j-i \equiv d$ $(\bmod n)$. We define a 0 -adjacency to be a singleton, that is, a block containing one element.

2010 AMS Mathematics subject classification. Primary 05A15, 05A18, 05A19.

Keywords and phrases. Adjacency, singleton, noncrossing partition, Catalan number.

Partially supported by the National Science Foundation grant No. 80860 .

Received by the editors on April 2, 2014, and in revised form on April 18, 2014. DOI:10.1216/RMJ-2016-46-1-301 
Callan [2] has proved that the number of singletons in all partitions of $A_{n}$ equals the number of 1-adjacencies, by giving a bijection in terms of an algorithm that interchanges singletons and 1-adjacencies.

We remark that the number of singletons in all partitions of $A_{n}$ is $n B_{n-1}$. This may be proved by fixing an index $j \in\{1, \ldots, n\}$, and noting that the number of partitions containing the singleton $\left\{a_{j}\right\}$ is $B_{n-1}$, which is the number of ways of inserting the block $\left\{a_{j}\right\}$ into a partition of $A_{n} \backslash\left\{a_{j}\right\}$.

The purpose of this note is to prove the following general result and establish a formula for the number of $d$-adjacencies in noncrossing partitions (for the latter, see Section 2).

Theorem 1.1. Let $n, d$ be integers, $0 \leq d<n$. Then the number of $d$-adjacencies in all partitions of $A_{n}$ is independent of $d$ and equal to $n B_{n-1}$.

The proof of Theorem 1.1 is a consequence of either of the following lemmas.

We will identify $A_{n}$ with the label set $[n]=\{1,2, \ldots, n\}$. Clearly, $\left(a_{i}, a_{j}\right)$ is a $d$-adjacency in a partition of $A_{n}$ if and only if $(i, j)$ is a $d$-adjacency in a partition of $[n]$.

Lemma 1.2. The number of d-adjacencies in all partitions of $[n]$ is $n B_{n-1}$ for all integers $0 \leq d \leq n-1$.

Proof. Since the number of singletons, or 0-adjacencies, is known to be $n B_{n-1}$, we consider the case $d>0$. There are precisely $n$ distinct $d$-adjacencies for each $d \in[n-1]$, namely,

$$
(a, a+d), \quad 1 \leq a \leq n-d \quad \text { with } \quad(n-d+c, c), 1 \leq c \leq d .
$$

Fix a $d$-adjacency $(a, a+d)(\bmod n), a \in[n]$. Note that the range of $a$ implies that $a+d \not \equiv 0(\bmod n)$. Then the number of partitions in which $a$ and $a+d(\bmod n)$ belong to the same block is given by $B_{n-1}$, which is obtained as the number of ways of partitioning the set $[n] \backslash\{a+d(\bmod n)\}$, followed by putting $a+d(\bmod n)$ into the block containing $a$. Hence, the result. 
The proof of the second lemma contains a solution to the problem, raised in $[\mathbf{1}, \mathbf{4}]$, of finding a bijection between 1-adjacencies and singletons.

Lemma 1.3. The multi-set of $d$-adjacencies in all partitions of $[n]$ is in one-to-one correspondence with the set of singletons in all partitions of $[n]$, for all integers $n, d, 1 \leq d<n$.

Proof. The type of bijection described below was popularized by Richard Stanley (see [5]). Here, "adjacency" means " $d$-adjacency."

We associate a partition of $[n]$ containing $m$ adjacencies with $m$ different partitions of $[n]$ containing singletons so that the number of times a given partition $\pi$ of $[n]$ appears is the same as the number of adjacencies in $\pi$.

Let $\pi$ be a partition of $[n]$ containing $m>0$ adjacencies. Write down $\pi$ a total of $m$ times, each corresponding to an adjacency. Then, for a fixed adjacency $x, x+d(\bmod n)$ the image of $\pi$ is obtained by creating a new singleton block containing $x+d(\bmod n)$, and then rearranging the blocks in standard order. For example, consider the partition $\pi=129 / 368 / 45 / 7$. When $d=1, \pi$ maps to $19 / 2 / 368 / 45 / 7,129 / 368 / 4 / 5 / 7$ and $1 / 29 / 368 / 45 / 7$, corresponding to the adjacencies $(1,2),(4,5)$ and $(9,1)$, respectively; when $d=2, \pi$ maps to $129 / 36 / 45 / 7 / 8$ and $19 / 2 / 368 / 45 / 7$, corresponding to the adjacencies $(6,8)$ and $(9,2)$, and so forth.

Conversely, delete each singleton $\{x\}$, and put $x$ into the block containing $x-d$ if $x>d$, or into the block containing $x+n-d$ if $x \leq d$. This gives the inverse image of a partition with respect to the singleton. For example, since it contains a singleton, the inverse image of $\pi=129 / 368 / 45 / 7$ is $129 / 3678 / 45$ when $d=1$, and $129 / 368 / 457$ when $d=2$.

This gives the desired bijection.

The full correspondence is illustrated for $n=4$ when $d=2$ in Table 1. As a verification of the inverse mapping observe that the number of occurrences of a partition in the third column is equal to the number of singletons the partition contains. 
TABLE 1. Bijection between 2-adjacencies and singletons for $n=4$.

\begin{tabular}{ccc} 
partition & 2-adjacency & image \\
\hline 1234 & 13 & $124 / 3$ \\
1234 & 24 & $123 / 4$ \\
1234 & 31 & $1 / 234$ \\
1234 & 42 & $134 / 2$ \\
$123 / 4$ & 13 & $12 / 3 / 4$ \\
$123 / 4$ & 31 & $1 / 23 / 4$ \\
$124 / 3$ & 24 & $12 / 3 / 4$ \\
$124 / 3$ & 42 & $14 / 2 / 3$ \\
$134 / 2$ & 13 & $14 / 2 / 3$ \\
$134 / 2$ & 31 & $1 / 2 / 34$ \\
$13 / 24$ & 13 & $1 / 24 / 3$ \\
$13 / 24$ & 24 & $13 / 2 / 4$ \\
$13 / 24$ & 31 & $1 / 24 / 3$ \\
$13 / 24$ & 42 & $13 / 2 / 4$ \\
$1 / 234$ & 24 & $1 / 23 / 4$ \\
$1 / 234$ & 42 & $1 / 2 / 34$ \\
$1 / 24 / 3$ & 24 & $1 / 2 / 3 / 4$ \\
$1 / 24 / 3$ & 42 & $1 / 2 / 3 / 4$ \\
$13 / 2 / 4$ & 13 & $1 / 2 / 3 / 4$ \\
$13 / 2 / 4$ & 31 & $1 / 2 / 3 / 4$ \\
\hline
\end{tabular}

2. Noncrossing partitions. A noncrossing partition of $[n]$ forbids the occurrence of four elements $w<x<y<z$ such that $w, y$ belong to one block and $x, z$ belong to another. Equivalently, a noncrossing partition is a partition of the vertices of a regular $n$-gon (labeled by $[n]$ and arranged clockwise on a circle) such that the convex hulls of its blocks are pairwise disjoint.

Denote the set of noncrossing partitions of $[n]$ by $N C(n)$. It is well known that

$$
|N C(n)|=C_{n}=\frac{1}{n+1}\left(\begin{array}{c}
2 n \\
n
\end{array}\right),
$$

where $C_{n}$ is the $n$th Catalan number.

We consider $N C(n)$ in the light of the correspondence established in Lemma 1.3.

It is not hard to see that 1-adjacencies and singletons are equidis- 
tributed in $N C(n)$, as already observed in [2]. This follows from the simple fact that connecting or disconnecting a pair of consecutive points on a circle cannot create a crossing.

However, the situation is different for $d$-adjacencies when $d>1$ : the (re-) connection of the member of a singleton $\{a\}$ to (the block containing) $a-d$, may create a crossing. For example, consider the inverse image of the noncrossing partition $\pi=129 / 368 / 45 / 7$, when $d=2$. The resulting partition is $129 / 368 / 457$ which is not noncrossing because of the integers $5,6,7,8$ with 5,7 in the third block and 6,8 in the second.

Denote by $y_{n}(d)$ the number of $d$-adjacencies in all noncrossing partitions of $[n]$. The following rotational symmetry relation obviously holds

$$
y_{n}(d)=y_{n}(n-d)
$$

It is also easy to show, as with unrestricted partitions, that

$$
y_{n}(0)=y_{n}(1)=n C_{n-1}=\left(\begin{array}{c}
2 n-2 \\
n-1
\end{array}\right) \text {. }
$$

The full formula is stated below.

\section{Theorem 2.1.}

$$
y_{n}(d)=n C_{d} C_{n-d}, \quad 1 \leq d \leq n-1
$$

Proof. We first count how many noncrossing partitions $\pi$ contain a certain $d$-adjacency $(a, a+d)$, for any $a \in[n], d \in[n-1]$.

The restriction of $\pi$ to $\{a+1, \ldots, a+d\}(\bmod n)$ is a noncrossing partition for which there are $C_{d}$ possibilities. Similarly, the restriction to $\{a+d+1, \ldots, n, 1, \ldots, a\}(\bmod n)$ is a noncrossing partition with $C_{n-d}$ possibilities.

This procedure can be reversed uniquely. Given a noncrossing partition of the set $\{a+1, a+2, \ldots, a+d\}$ and another noncrossing partition of the set $\{a+d+1, \ldots, a\}$, combine them by merging the blocks containing $a$ and $a+d$. By construction this merging process cannot create a crossing. 
Hence, there are precisely $C_{d} C_{n-d}$ noncrossing partitions containing the $d$-adjacency $(a, a+d)$. Since there are $n$ possible choices for $a$, this gives a total of $n C_{d} C_{n-d} d$-adjacencies.

It follows from the Catalan-number recurrence

$$
C_{0}=1, \quad C_{n+1}=\sum_{j=0}^{n} C_{j} C_{n-j}
$$

that

$$
\sum_{d=1}^{n-1} y_{n}(d)=n C_{n+1}-2 n C_{n}=2\left(\begin{array}{c}
2 n \\
n-2
\end{array}\right) .
$$

We remark that Theorem 2.1 gives a seemingly new interpretation of the $j$ th summand in (2.3) as the number of $j$-adjacencies $(a, a+j)$ in all noncrossing partitions of $[n]$, for each $a \in[n]$.

Lastly, we recall Stirling's asymptotic approximation of the factorial function:

$$
n ! \sim \sqrt{2 \pi n} e^{-n} n^{n}
$$

where the standard notation $\sim$ is defined by $u \sim v$ if and only if $\lim _{n \rightarrow \infty} u / v=1$. Using (2.4) and the Catalan-number formula (2.1) one can show that

$$
C_{n} \sim \frac{4^{n}}{\sqrt{\pi n^{3}}}
$$

Consequently,

$$
y_{n}(d) \sim \frac{4^{n}}{\pi \sqrt{d^{3} n}}
$$

We can now state:

Theorem 2.2. Given positive integers $n$ and $d, 0<d<n$, the average number of $d$-adjacencies in a random noncrossing partition of $[n]$ is given by

$$
\frac{n C_{d} C_{n-d}}{C_{n}}=\frac{n(n+1)}{(d+1)(n-d+1)}\left(\begin{array}{c}
2 d \\
d
\end{array}\right)\left(\begin{array}{c}
2(n-d) \\
n-d
\end{array}\right)\left(\begin{array}{c}
2 n \\
n
\end{array}\right)^{-1} \sim \frac{n}{\sqrt{\pi d^{3}}}
$$


Proof. The first equality follows from (2.1). The asymptotic part may be obtained by using the exact formula in the theorem together with (2.4):

$$
\frac{n C_{d} C_{n-d}}{C_{n}} \sim \frac{n 4^{d}}{\sqrt{\pi d^{3}}} \frac{4^{n-d}}{\sqrt{\pi(n-d)^{3}}} \frac{\sqrt{\pi n^{3}}}{4^{n}}=\frac{n \sqrt{\pi n^{3}}}{\sqrt{\pi^{2} d^{3}(n-d)^{3}}},
$$

which, for large $n$, is the same as

$$
\frac{n \sqrt{\pi n^{3}}}{\sqrt{\pi^{2} d^{3} n^{3}}}
$$

Acknowledgments. The author thanks Stephan Wagner for suggesting the proof of Theorem 2.1.

\section{REFERENCES}

1. F.R. Bernhart, Catalan, Motzkin, and Riordan numbers, Discr. Math. 204 (1999), 73-112.

2. D. Callan, On conjugates for set partitions and integer compositions, arXiv.math.CO/0508052.

3. L. Comtet, Advanced combinatorics. The art of finite and infinite expansions, D. Reidel Publishing Co., 1974.

4. W.Y.C. Chen and D.G.L. Wang, On singletons and adjacencies of set partitions of type B, Discr. Math. 311 (2011), 418-422.

5. R. Stanley, Enumerative combinatorics, Volume 1, Cambridge University Press, New York, 1997.

School of Mathematics, University of the WitwatersRand, Wits 2050, Johannesburg, South Africa

Email address: Augustine.Munagi@wits.ac.za 